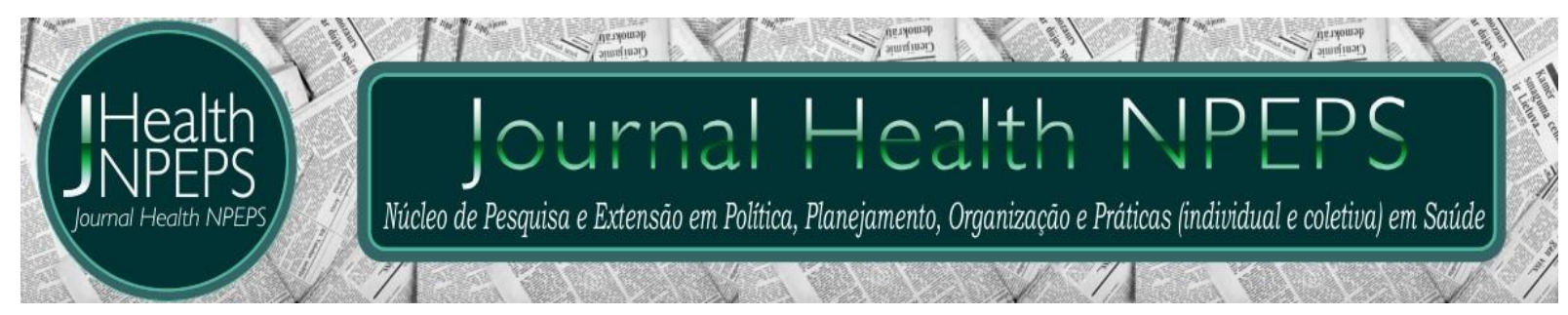

ARTIGO ORIGINAL

\title{
Sistematização da assistência de enfermagem no monitoramento clínico de pacientes com hantavirose
}

\section{The systematization of nursing care in the clinical monitoring of patients with hantavirus}

\section{La sistematización de los cuidados de enfermería en el seguimiento clínico de los pacientes con hantavirus}

\section{Ana Cláudia Pereira Terças ${ }^{1}$, Ingrid Gomes de Souza ${ }^{2}$, Ariadne Cristinne Pereira de Moura $^{3}$, Vagner Ferreira do Nascimento ${ }^{4}$, Thalise Yuri Hattori ${ }^{5}$, Josué Souza Gleriano $^{6}$, Angélica Pereira Borges ${ }^{7}$, Juliana Herrero da Silva ${ }^{8}$}

\begin{abstract}
RESUMO
Objetivo: aplicar a sistematização da assistência de enfermagem para pacientes com Síndrome Pulmonar por Hantavírus. Método: estudo quanti-qualitativo, observacional e prospectivo sobre acompanhamento clínico de paciente com hantavirose atendidos em hospital de referência do médio norte mato-grossense. Os dados foram coletados no ano de 2014, com cinco pacientes hospitalizados. As intervenções de enfermagem foram pautadas nos diagnósticos de NANDA identificados e utilizou-se as recomendações da CIPE. Resultados: a maioria dos acometidos são do sexo masculino, de cor branca, presente em várias faixas etárias, com residência e/ou atividades profissionais realizadas em zona rural. Dentre os diagnósticos destacou: troca de gases prejudicada, padrão respiratório ineficaz, volume de líquido excessivo, processos familiares interrompidos e risco de infecção. Conclusão: a aplicação da sistematização à uma doença de alta letalidade e de rápida evolução clínica é essencial para fornecer um cuidado organizado, ético e baseado em evidências científicas que irão subsidiar a qualidade da assistência prestada.
\end{abstract}

\footnotetext{
${ }^{1}$ Enfermeira. Doutora em Medicina Tropical. Docente Adjunta da Universidade do Estado do Mato Grosso. Campus Tangará da Serra. Departamento de Enfermagem. Pesquisadora Laboratório de Hantaviroses e Rickettsioses, Instituto Oswaldo Cruz - FIOCRUZ/RJ. E-mail: ana.claudia@unemat.br Autora principal - Endereço para correspondência: Rua José Garcia Lacerda, 152N, Centro Tangará da Serra - MT - Brasil.

${ }^{2}$ Acadêmica de Medicina da Universidade do Estado do Mato Grosso. Cáceres - MT - Brasil. E-mail: ingrid.souza@hotmail.com

${ }^{3}$ Acadêmica de Enfermagem da Universidade do Estado do Mato Grosso. Campus de Tangará da Serra. Tangará da Serra - MT - Brasil. E-mail: ariadnecristinnemoura@hotmail.com

${ }^{4}$ Enfermeiro. Doutorando em BIOÉTICA. Docente Assistente da Universidade do Estado do Mato Grosso. Campus Tangará da Serra. Departamento de Enfermagem. Tangará da Serra - MT - Brasil. E-mail: vagnerschon@hotmail.com ${ }^{5}$ Enfermeira. Mestre em Ciências da Saúde. Docente Assistente da Universidade do Estado do Mato Grosso. Campus Tangará da Serra. Departamento de Enfermagem. Tangará da Serra - MT - Brasil. E-mail: thalisehattori@gmail.com ${ }^{6}$ Enfermeiro. Mestre em Saúde Coletiva. Docente Assistente da Universidade do Estado do Mato Grosso. Campus Tangará da Serra. Departamento de Enfermagem. Tangará da Serra - MT - Brasil. E-mail: josue_gleriano@hotmail.com ${ }^{7}$ Enfermeira. Mestre em Enfermagem. Docente Assistente da Universidade do Estado do Mato Grosso. Campus Tangará da Serra. Departamento de Enfermagem. Tangará da Serra - MT - Brasil. E-mail: angel.ufmt@gmail.com

${ }^{8}$ Enfermeira. Mestranda em Genética e Biologia Molecular pela Universidade Federal do Rio Grande do Sul. Coordenadora da Vigilância Epidemiológica, Prefeitura Municipal de Tangará da Serra. Tangará da Serra - MT - Brasil. E-mail: epidemio@tangaradaserra.mt.gov.br
} 
Descritores: Hantavírus; Enfermagem; Cuidados de Enfermagem.

\begin{abstract}
Objective: apply the systematization of nursing care for patients with Hantavirus Pulmonary Syndrome. Method: quantitative, observational and prospective study on clinical follow-up of a patient with hantavirosis treated at a referral hospital in the northern mato-grossense region. Data were collected in 2014, with five hospitalized patients. Nursing interventions were based on the identified NANDA diagnoses and CIPE recommendations were used. Results: the majority of the patients are white, male, present in several age groups, with residence and/or professional activities carried out in rural areas. Among the diagnoses highlighted: impaired gas exchange, ineffective breathing pattern, excessive fluid volume, interrupted family processes and risk of infection. Conclusion: the application of systematization to a disease of high lethality and rapid clinical evolution is essential to provide an organized, ethical and evidencebased care that will subsidize the quality of care provided.
\end{abstract}

Descriptors: Hantavirus; Nursing; Nursing care.

\title{
RESUMEN
}

Objetivo: aplicar la sistematización de la asistencia de enfermería para pacientes con Síndrome Pulmonar por Hantavirus. Método: estudio cuantitativo y cualitativo, observacional y prospectivo en pacientes seguimiento clínico con hantavirus atendidos en un hospital de referencia en el norte de Mato Grosso promedio. Los datos fueron recogidos en 2014, con cinco pacientes hospitalizados. Las intervenciones de enfermería se guiaron por los diagnósticos NANDA identificados y se utilizan las recomendaciones de la CIPE. Resultados: los más afectados son masculinos, blancos, presentes en diversos grupos de edad, con domicilio y / o actividades profesionales en las áreas rurales. Entre los diagnósticos dicho: intercambio de gases perjudicado, patrón respiratorio ineficaz, el volumen de exceso de líquido, los procesos familiares interrumpidas y el riesgo de infección. Conclusión: la aplicación de sistematización en una enfermedad altamente letal y rápido curso clínico es esencial para proporcionar una atención organizada, ética y basada en la evidencia científica que apoye la calidad de la atención.

Descriptores: Hantavirus; Enfermería; Cuidados de enfermería.

\section{INTRODUÇÃO}

A Síndrome Pulmonar por Hantavírus (SPH) é uma doença viral, emergente e aguda que causa grande impacto nas Américas ${ }^{1}$. É causada pelo hantavírus que se encontra associado a roedores silvestres americanos da família Cricetidae e sub- família Sigmodontinae ${ }^{2}$. A transmissão se inicia quando ocorre a infecção do homem por meio da inalação de aerossóis de inócuos virais provenientes de excretas e secreções, presentes no ambiente contaminado 3 .

A doença é apresentada por quatro fases de manifestações clínicas: inicial ou prodrômica, cardiopulmonar, diurética e convalescência e gera grande preocupação em 
virtude do potencial virulento e da rápida evolução com que causa os óbitos, em média 24 horas após o início dos sintomas ${ }^{4}$.

No estado de Mato Grosso, os primeiros casos ocorreram em 1999, no município de Campo Novo do Parecis, sendo registrados 203 casos até 2010. Letalidade esta que chega a 42,8\%. Estudos ecoepidemiológicos na região do médio norte e nos municípios de abrangência da BR 163 identificaram os seguintes reservatórios e as respectivas variantes virais: Oligoryzomys utiairitensis (Castelo dos Sonhos) e Calomys callidus (Laguna Negra). A região do médio norte de Mato Grosso, cujo polo regional de saúde se localiza em Tangará da Serra, é responsável por 71,9\% dos casos do Estado ${ }^{4,5}$.

Para atender essas notificações, leva-se em consideração a gravidade que cada caso requer, sendo importante a qualificação da equipe de saúde para a prestação de uma assistência garantida pela excelência, baseada em evidências clínicas e científicas.

Nessa perspectiva, o cuidado de enfermagem é primordial por contribuir decisivamente nesse processo, onde o sucesso do tratamento paralelamente aos aspectos epidemiológicos e características individuais dos sujeitos, deve-se às condutas e práticas terapêuticas desenvolvidas. Para a execução e gerenciamento dessas atividades, os enfermeiros utilizam a sistematização da assistência em enfermagem (SAE), entendida como uma atividade privativa desse profissional, com a finalidade de identificar situações de saúde e doença dos indivíduos por meio de uma estratégia metodológica científica, direcionado a um cuidado seguro e global na ótica da promoção, prevenção, recuperação e reabilitação da saúde do cliente $e^{6,7}$.

Os modelos teóricos têm contribuído de forma substancial no processo de trabalho da enfermagem quando utilizados como referencial para a SAE, pois proporcionam meios para organizar as informações coletadas no histórico, analisar e interpretar esses dados, além de intervir e avaliar os resultados do cuidado implementado7 ${ }^{7}$. Assim, na realidade deste estudo, optou-se por construir os diagnósticos e intervenções de enfermagem tendo como referencial a Teoria das Necessidades Humanas Básicas proposta por Wanda Horta ${ }^{8}$.

A implementação da SAE nos pacientes com SPH é de extrema relevância, devido à complexidade dos cuidados e pelas habilidades especiais que a equipe de enfermagem deve possuir durante a abordagem inicial e ao longo do tratamento.

Ressalta-se então a importância de sistematizar a assistência de enfermagem, pois este instrumento norteará as condutas do enfermeiro nas situações de 
gerenciamento da equipe, o que poderá resultar em um cuidado individualizado e humanizado, refletindo positivamente na qualidade da assistência bem como na redução da mortalidade.

Assim, objetivou-se descrever a aplicação da SAE aos pacientes com SPH em um hospital de referência localizado na região médio norte de Mato Grosso.

\section{MÉTODO}

Trata-se de um estudo transversal e descritivo sobre o acompanhamento clínico de pacientes com hantavirose. A população do estudo foi composta por cinco pacientes com SPH, hospitalizados em instituição privada de saúde, que possuem leitos conveniados do Sistema Único de Saúde (SUS), localizado na região do médio norte de Mato Grosso no período de agosto a dezembro de 2014.

Esse serviço de saúde é referência regional para atender pacientes que possam necessitar de Unidade de Terapia Intensiva (UTI), além de possuir profissionais habilitados e com experiência em atender pacientes com suspeita de hantavirose. Ressalta-se ainda que neste serviço não existe protocolo de atendimento, nem SAE implementada para essa clientela.

Dessa forma, a dinâmica da pesquisa iniciou após a notificação compulsória e imediata enviada ao setor de vigilância epidemiológica do município, com a realização do levantamento de dados que compuseram o histórico de enfermagem de todos os pacientes. Esta atividade se baseou na entrevista com paciente e profissionais de saúde, seguido da realização do exame físico e preenchimento da ficha de notificação/investigação. Ressalta-se que a coleta de dados ocorreu no primeiro dia de hospitalização.

Todas as informações do histórico dos pacientes suspeitos de hantavirose são arquivadas pelo serviço de vigilância local rotineiramente, porém, a necessidade de se ampliar as discussões e dar cientificidade as ações da enfermagem, possibilitaram a parceria entre os serviços de saúde e a universidade local para aplicação da SAE a esses pacientes.

A elaboração dos diagnósticos e intervenções de enfermagem foi construído para todos os pacientes suspeitos em atendimento, uma vez que a notificação acontece com a suspeita clínica que é confirmada através da realização de sorologia e detecção da 
presença de anticorpos do tipo IgM, assim os pacientes sem confirmação laboratorial foram excluídos do estudo.

Em decorrência da SAE ser utilizada como estrutura do processo de enfermagem para organização das informações, composto pelo histórico, diagnóstico de enfermagem, planejamento, intervenções de enfermagem e avaliação, torna-se primordial que todas as etapas sejam realizadas. Porém, neste estudo não foi realizada a etapa de avaliação, pois a doença apresenta rápida evolução, inclusive com óbito em três dos pacientes acompanhados.

Os diagnósticos de enfermagem foram construídos pela equipe utilizando-se a taxonomia da NANDA (North American Nursing Diagnosis Association) ${ }^{9}$ e as intervenções de enfermagem foram pautadas na CIPE (Classificação Internacional para a Prática de Enfermagem $)^{10}$. No trabalho, optou-se por discutir os diagnósticos e as intervenções de enfermagem prioritárias comum a todos os casos.

Como forma de manter o sigilo e a privacidade dos participantes, foi adotada a identificação dos sujeitos, utilizando codificação do tipo alfanumérica, de modo que a letra $\mathrm{C}$ indica cliente, e o elemento numérico que compõe o conjunto apenas indica a ordem de avaliação.

Todos os aspectos éticos exigidos em pesquisa com seres humanos (Resolução 466/12) foram respeitados, o estudo faz parte do projeto intitulado "Consulta de Enfermagem no monitoramento clínico da hantavirose" com Certificado para Apresentação de Apreciação Ética (CAAE) 28569414.8.1001.5166 e aprovação com número de parecer 684.584 por meio do Comitê de Ética em Pesquisa (CEP) da Universidade do Estado de Mato Grosso.

\section{RESULTADOS}

0 perfil dos pacientes atendidos no serviço de referência do médio norte de Mato Grosso, como observado na Tabela 1, apresentou maioria do sexo masculino (80\%), de cor branca (60\%), presente em todas as faixas etárias (11-62 anos), com residência e/ou atividades profissionais realizadas em zona rural (100\%).

Tabela 1 - Características pessoais, clínicas, laboratoriais e terapêuticas de pacientes com SPH na região médio norte de Mato Grosso. 2014. Médio Norte de Mato Grosso, Brasil.

\begin{tabular}{lrr}
\hline \multicolumn{1}{c}{ Variáveis } & \multicolumn{2}{c}{ Total } \\
\cline { 2 - 3 } & N & $\%$ \\
\hline Journal Health NPEPS. 2017; 2(2):391-406. & 395
\end{tabular}




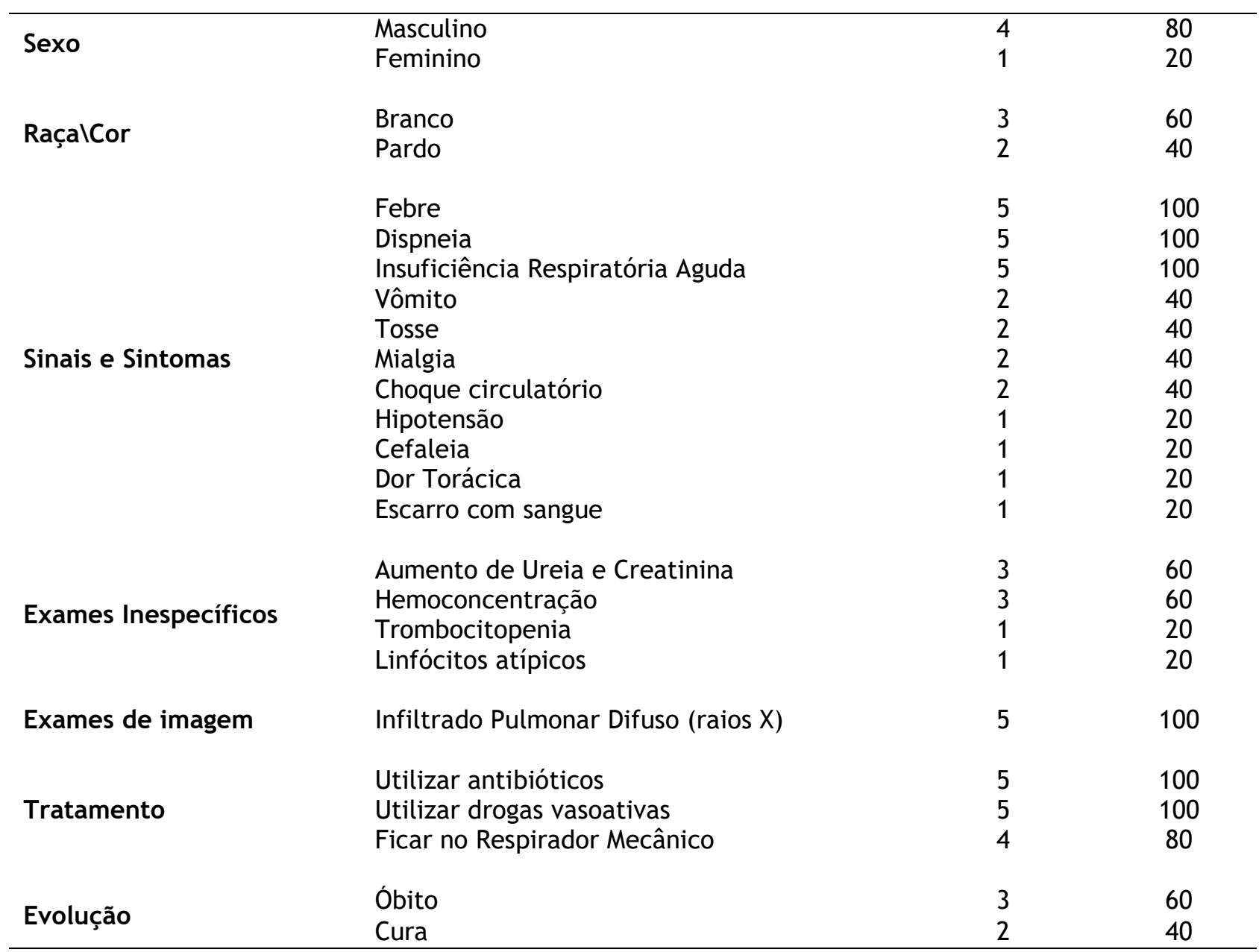

Onze diferentes sinais e sintomas foram evidenciados nos pacientes deste estudo, porém a presença de escarro com sangue em um dos pacientes chamou a atenção, pois o mesmo não é descrito em pacientes com SPH. Com relação aos exames laboratoriais inespecíficos e de imagem foi identificado em nosso estudo a sua realização, bem utilização de terapêutica inespecífica e destaque para letalidade que atingiu $60 \%$.

A coleta de dados forneceu subsídios para a identificação de 22 diferentes diagnósticos de enfermagem aos pacientes com hantavirose com média de 11 diagnósticos por pacientes.

Dentre os diagnósticos foram elencados cinco diagnósticos prioritários, pois os mesmos foram identificados em todos os clientes, sendo eles: troca de gases prejudicada, padrão respiratório ineficaz, volume de líquido excessivo, processos familiares interrompidos e risco de infecção. No Quadro 1 são apresentados os diagnósticos reais, seus fatores relacionados e suas características definidoras. 
Quadro 1 - Diagnósticos de enfermagem reais (NANDA 2015-2017) para pacientes com SPH da região médio norte de Mato Grosso, 2015.

\begin{tabular}{|c|c|c|}
\hline $\begin{array}{l}\text { Diagnóstico de } \\
\text { Enfermagem }\end{array}$ & Fatores Relacionados & Características Definidoras \\
\hline $\begin{array}{l}\text { Troca de Gases } \\
\text { Prejudicada }\end{array}$ & $\begin{array}{l}\text { Desequilíbrio na relação } \\
\text { ventilação - perfusão }\end{array}$ & $\begin{array}{l}\text { - Dispneia } \\
\text { - Padrão respiratório anormal; } \\
\text { - Hipóxia; } \\
\text { - Gases sanguíneos arteriais } \\
\text { anormais; }\end{array}$ \\
\hline $\begin{array}{l}\text { Volume de Líquido } \\
\text { Excessivo }\end{array}$ & $\begin{array}{l}\text { Mecanismos reguladores } \\
\text { comprometidos } \\
\text { (extravasamento de líquido } \\
\text { para espaço intersticial) }\end{array}$ & $\begin{array}{l}\text { - Dispneia; } \\
\text { - Edema; } \\
\text { - Congestão Pulmonar; } \\
\text { - Alteração no padrão respiratório. }\end{array}$ \\
\hline $\begin{array}{l}\text { Padrão Respiratório } \\
\text { Ineficaz }\end{array}$ & $\begin{array}{l}\text {-Síndrome da hipoventilação } \\
\text { (infiltração pulmonar); } \\
\text { - Fadiga }\end{array}$ & $\begin{array}{l}\text { - Dispneia } \\
\text { - Padrão respiratório anormal } \\
\text { (ventilação mecânica) }\end{array}$ \\
\hline $\begin{array}{l}\text { Processos Familiares } \\
\text { Interrompidos }\end{array}$ & $\begin{array}{l}\text { - Mudanças do estado de } \\
\text { saúde de um membro da } \\
\text { família; } \\
\text { - Mudança na interação com } \\
\text { comunidade; } \\
\text { - Transição situacional; }\end{array}$ & $\begin{array}{l}\text { Mudanças no padrão de } \\
\text { relacionamento decorrente da } \\
\text { hospitalização. }\end{array}$ \\
\hline
\end{tabular}

Outro diagnóstico prevalente nesses indivíduos foi o risco de infecção que NANDA $^{9}$ define como vulnerabilidade à invasão e multiplicação de organismos patogênicos, que podem comprometer a saúde.

Quadro 2 - Diagnósticos de enfermagem (NANDA 2015-2017) de risco para pacientes com SPH da região médio norte de Mato Grosso, 2015.

\begin{tabular}{|l|l|}
\hline $\begin{array}{l}\text { Diagnóstico de } \\
\text { Enfermagem }\end{array}$ & \multicolumn{1}{|c|}{ Fatores de Risco } \\
\hline Risco de Infecção & $\begin{array}{l}\text { Exposição ambiental aumentada a patógenos (decorrente } \\
\text { da hospitalização); }\end{array}$ \\
$-\begin{array}{l}\text { Procedimentos invasivos (punção venosa, sondagem } \\
\text { vesical de demora, oxigenioterapia, ventilação } \\
\text { mecânica); } \\
\text { Defesas primárias inadequadas com alteração na } \\
\text { integridade da pele (punção venosa). }\end{array}$ \\
\hline
\end{tabular}

As intervenções de enfermagem foram pautadas nos cinco diagnósticos prioritários identificados e utilizou-se as recomendações da CIPE $^{11}$ para sua construção. 0 quadro 3 apresenta as intervenções propostas de acordo com os diagnósticos de enfermagem identificados.

Quadro 3 - Intervenções de enfermagem (CIPE) por diagnóstico prioritário para pacientes com SPH da região médio norte de Mato Grosso, 2015.

\begin{tabular}{|l|l|}
\hline $\begin{array}{l}\text { Diagnóstico de } \\
\text { Enfermagem }\end{array}$ & \multicolumn{1}{c|}{ Intervenções de Enfermagem } \\
\hline $\begin{array}{l}\text { Troca de Gases } \\
\text { Prejudicada }\end{array}$ & $\begin{array}{l}\text { - Colocar o paciente na posição fowler alta ou semi fowler; } \\
\text { - Fornecer oxigênio para evitar e prevenir hipoxia e dispneia; }\end{array}$ \\
\hline
\end{tabular}




\begin{tabular}{|c|c|}
\hline & $\begin{array}{l}\text { - Auscultar sons respiratórios; } \\
\text { - Monitorar sinais vitais de 4/4 horas; } \\
\text { - Prevenir choque; } \\
\text { - Monitorar resultado de gasometria arterial; } \\
\text { - Aspirar secreções para manter as vias aéreas permeáveis. }\end{array}$ \\
\hline $\begin{array}{l}\text { Volume de } \\
\text { Liquido } \\
\text { Excessivo }\end{array}$ & $\begin{array}{l}\text { - Controlar diurese das } 24 \text { horas quanto ao volume e } \\
\text { características; } \\
\text { - Fazer controle rigoroso de infusões venosas; } \\
\text { - Registrar ingestão e excretas de líquidos; } \\
\text { - Avaliar a presença de edema no corpo, diariamente; } \\
\text { - Prevenir choque; } \\
\text { - Monitorizar sons respiratórios, gasometria arterial e eletrólitos; } \\
\text { - Monitorizar ventilador mecânico. }\end{array}$ \\
\hline $\begin{array}{l}\text { Padrão } \\
\text { Respiratório } \\
\text { Ineficaz }\end{array}$ & $\begin{array}{l}\text { - Manter o paciente levemente desidratado recebendo de } 1 \text { a 1,5 } \\
\text { litros de soro a cada } 24 \text { h; } \\
\text { - Fazer o controle da ingesta hídrica, observar e controlar o } \\
\text { gotejamento de infusão de eletrólitos e se atentar ao } \\
\text { aparecimento de edemas; } \\
\text { - Auscultar sons respiratórios; } \\
\text { - Monitorar sinais vitais de } 4 / 4 \text { horas; } \\
\text { - Prevenir choque; } \\
\text { - Monitorizar ventilador mecânico. }\end{array}$ \\
\hline $\begin{array}{l}\text { Processos } \\
\text { Familiares } \\
\text { Interrompidos }\end{array}$ & $\begin{array}{l}\text { - Oferecer a assistência até que o paciente esteja totalmente } \\
\text { capacitado a assumir o autocuidado; } \\
\text { - Orientar a família/cuidador da importância de estimular o } \\
\text { autocuidado. } \\
\text { - Observar sentimentos de tristeza, irritabilidade, medo, } \\
\text { ansiedade e solidão, buscando subsídios para compreender o } \\
\text { estado emocional do paciente e familiares para possibilitar-lhes } \\
\text { apoio. } \\
\text { - Oferecer apoio psicológico; } \\
\text { - Oferecer informações sobre o diagnóstico, tratamento e } \\
\text { prognóstico; } \\
\text { - Esclarecer todas as dúvidas da família; } \\
\text { - Incentivar a família quanto a sua importância na recuperação do } \\
\text { indivíduo. }\end{array}$ \\
\hline $\begin{array}{l}\text { Risco de } \\
\text { Infecção }\end{array}$ & $\begin{array}{l}\text { - Avaliar o estado nutricional; } \\
\text { - Avaliar locais de inserção de cateteres quanto à presença de } \\
\text { hipertermia; } \\
\text { - Controlar os líquidos e eletrólitos através de balanço hídrico; } \\
\text { - Monitorar temperatura e frequência respiratória; } \\
\text { - Utilizar técnicas assépticas no manuseio do paciente; } \\
\text { - Avaliar padrão respiratório e ventilador mecânico; } \\
\text { - Realizar higiene corporal e do couro cabeludo no leito. }\end{array}$ \\
\hline
\end{tabular}

\section{DISCUSSÃO}

A SPH atinge a maioria dos países das Américas, tendo como característica a ocorrência de casos humanos em todas as faixas etárias, em ambos os sexos e presente nas diferentes classes sociais, sendo comum a todas as localidades principalmente 0 contato com a zona rural, fato este que está relacionado com a necessidade do contato com as excretas de roedores silvestres para se estabelecer o ciclo de transmissão. 0 
perfil americano destaca maior incidência na faixa etária produtiva e em homens com atividades profissionais ou pessoais em área rural ou silvestre ${ }^{11,12}$.

Os achados aqui descritos sobre o perfil dos pacientes corrobora com os casos norte americanos e com estudo anterior de casos de hantavirose em Mato Grosso no período de 1999 a 2010 em que se identificou que os pacientes eram em sua maioria do sexo masculino (75,4\%), a idade variou entre 4 e 70 anos, com média de 30,53 anos e maior concentração na faixa etária entre 20 e 39 anos (53,7\%), tendo como raça/cor predominante a branca $(49,2 \%)$ e residência em zona rural $(56,1 \%)^{5}$.

A alta porcentagem de sinais e sintomas inespecíficos, como febre, cefaleia e mialgia mostra que a doença tem um início inespecífico e de difícil diagnóstico. Porém, a presença de tosse e dispneia na totalidade dos casos demonstra que houve predominância de quadro clínico de evolução grave, desencadeado pela alta taxa de hospitalização e da necessidade de assistência respiratória mecânica. Esses sinais e sintomas dos casos de Mato Grosso não diferem dos apresentados em outros estudos realizados no Brasil e nas Américas ${ }^{13-15}$.

As alterações hematológicas evidenciadas em exames inespecíficos realizados no primeiro dia de hospitalização dos pacientes apresentaram dados compatíveis com estudos já conduzidos em Mato Grosso, com hemoconcentração atingindo 60\% e presença de linfócitos atípicos em 20\%5,16. Em comparação com esses mesmos estudos foram encontradas divergências, uma delas se refere ao aumento de ureia e creatinina (60\%), pois nos demais estudos a prevalência dessa alteração laboratorial não era detectada em mais de $40 \%$ dos pacientes, já a trombocitopenia foi identificada com maior frequência nos outros estudos com uma média de $60 \%$ dos pacientes, já nos cinco pacientes acompanhados a frequência não superou os $20 \%$.

Em decorrência de todos os pacientes apresentarem infiltrado pulmonar difuso ao exame de raios $X$, pode ser uma evidência que todos já estavam na fase cardiopulmonar da doença, com comprometimento pulmonar e em sua maioria necessitavam de suporte ventilatório (80\%). Quanto ao tratamento, atualmente não existem antivirais ou outro tipo de tratamento que cure a SCPH. Na fase prodrômica deve ser realizado o monitoramento dos sinais vitais e da evolução da doença ${ }^{3}$ e a partir do início da fase cardiopulmonar os pacientes devem ser admitidos imediatamente em centros de terapia intensiva, para manutenção da vida e tratamento sintomático. Observa-se que a administração de drogas vasoativas e antibióticos foram realizadas em 
todos os pacientes.

A permanência hospitalar variou de dois a nove dias (média de cinco dias), sendo que C1 permaneceu por dois dias, não necessitando de ventilação mecânica e nem de suporte de UTI, evoluindo para cura, bem como C5 que também evoluiu para cura com alta hospitalar no quinto dia, porém o mesmo necessitou de assistência em UTI.

Deste modo, C2, C3, C4 e C5 foram hospitalizados na UTI e submetidos a ventilação mecânica, sendo que o C2 faleceu após nove dias de hospitalização, C3 evoluiu para óbito em seis dias e C4 morreu no terceiro dia, fato este que corrobora com a alta letalidade e evolução clínica rápida para óbito característicos da doença3,5.

Pelo fato da patogênese do hantavírus ter início nos pulmões, os pacientes apresentaram diversos diagnósticos relacionados ao padrão respiratório, devido ao tropismo que o mesmo apresenta pelas células endoteliais dos pulmões, fazendo com que a oferta de oxigênio seja insuficiente para suprir as necessidades vitais ${ }^{2,3}$.

O diagnóstico de troca de gases prejudicada é definido como excesso ou déficit na oxigenação e/ou na eliminação de dióxido de carbono na membrana alveolocapilar. Já o diagnóstico de padrão respiratório ineficaz é definido como inspiração e/ou expiração que não proporcionam ventilação adequada?.

A troca de gases prejudicada nos pacientes com hantavírus e o padrão respiratório ineficaz podem ser explicados pelo fato da infecção começar nos pulmões, nas células do endotélio onde a penetração é mediada por um complexo das integrinas B3. Logo em seguida, o vírus infecta os macrófagos alveolares, alojando-se posteriormente nos grandes órgãos, como fígado, coração, baço, rim e nódulos linfáticos. Este processo resulta no aumento da permeabilidade vascular e, consequentemente, no extravasamento de líquidos para o espaço intersticial desses órgãos, esse extravasamento é evidenciado através do raio-x no qual $100 \%$ dos pacientes apresentaram infiltrado pulmonar difuso ${ }^{2,14,17 .}$

A dispneia e a insuficiência respiratória são fatores presentes em todos os pacientes analisados e estas duas manifestações ocorrem devido à saída de proteínas para o parênquima pulmonar, por meio das alterações na permeabilidade do endotélio, resultando em um desequilíbrio da relação ventilação - perfusão, exigindo hospitalização de caráter imediato, com a necessidade de ventilação mecânica ${ }^{3}$.

Como consequência do aumento da permeabilidade endotelial, ocorre a congestão pulmonar que é caracterizada pelo diagnóstico denominado volume de líquido 
excessivo e definido como retenção aumentada de líquidos isotônicos ${ }^{9}$. É um diagnóstico característico em pacientes com hantavirose devido ao infiltrado pulmonar, a este diagnóstico se ressalta a importância do monitoramento constante da equipe de enfermagem quanto à oferta hídrica oferecida ao paciente, que deve ser baseada em um balanço hídrico rigoroso somente para suprir as necessidades celulares do indivíduo, pois o excesso de infusão de líquidos pode agravar o caso podendo o mesmo, evoluir para óbito precocemente ${ }^{2,3}$.

Um aspecto que não pode ser esquecido para esses pacientes é o processo familiar interrompido, que é definido como uma mudança nos relacionamentos e/ou no funcionamento da família9. Esta interrupção dos processos familiares é intermediada pela comunicação prejudicada que os pacientes apresentam. 0 contato da família com o paciente é extremamente significativo para o bem-estar, assim como também influencia no processo saúde-doença, exigindo da equipe de enfermagem a inserção da família no plano de cuidado, pois a mesma está diretamente relacionada à qualidade de vida do paciente ${ }^{18,19}$.

Este fator está diretamente relacionado à submissão do paciente a diversos procedimentos invasivos, o que consequentemente, aumenta a sua suscetibilidade de contrair uma infecção. 0 uso de cateteres intravenosos é indispensável na UTI, porém são considerados importantes fontes de infecção da corrente sanguínea primária, caso sejam manipulados de forma inadequada ${ }^{20}$. 0 que enfatiza a importância da equipe de enfermagem em realizar a manutenção do acesso venoso de forma criteriosa, minimizando os riscos de se tornar uma potente fonte de infecção.

Frente a esses diagnósticos prioritários, faz-se então necessário a elaboração das intervenções de enfermagem que foram planejadas de acordo com o Quadro 3 apresentado acima.

A hantavirose possui uma rápida evolução e os sintomas iniciais são inespecíficos como, cefaléia, astenia, febre, podendo ocorrer também náuseas e vômitos. No terceiro dia, o paciente apresenta tosse seca que posteriormente se torna produtiva e dispneia, que inicialmente é de leve intensidade, mas que na maioria dos casos, em menos de 24 horas, evolui para insuficiência respiratória grave, necessitando muitas vezes da instituição de suporte ventilatório ${ }^{3}$.

Em geral os pacientes com alterações respiratórias recebem oxigenoterapia para elevar a pressão arterial de oxigênio visando retorná-la a linha basal normal do paciente, 
a qual pode variar de 60 a $95 \mathrm{mmHg}$. O oxigênio corrigirá a agitação, confusão e agressividade que podem ser provocadas pela hipóxia cerebral. A administração de oxigenoterapia e a efetividade da ventilação mecânica são medidas críticas para a sobrevivência assim como manter o paciente em posição de fowler alta ou semi fowler para favorecer a mecânica respiratória diminuindo o consumo de 02 por meio da limitação dos movimentos ${ }^{21}$.

A elevação do tórax do paciente, em condições hemodinâmicas estáveis, acentua a ação da gravidade sobre as secreções das periferias pulmonares, drenando-as para a árvore brônquica, nos ramos mais calibrosos de onde podem ser aspirados ou eliminados pela tosse além de promover o repouso do paciente ${ }^{22}$. Nessa síndrome, a resposta imune exagerada é a principal responsável pela gravidade da doença ocasionando a infiltração no tecido pulmonar por linfócitos $\mathrm{TCD}_{8}$ que desencadeia edema pulmonar e insuficiência respiratória aguda ${ }^{2,23}$. No tratamento do edema agudo de pulmão é fundamental aspirar as secreções para manter as vias aéreas permeáveis além de monitorizar sons respiratórios, gasometria arterial e eletrólitos ${ }^{21}$.

Pacientes com hantavirose devem ser mantidos levemente desidratados, recebendo de 1 a 1,5 litros de soro a cada $24 \mathrm{~h}$, pois o excesso de líquidos poderá agravar o edema pulmonar. Fazer o controle da ingestão hídrica, observar e controlar o gotejamento de infusão de eletrólitos e atentar-se ao aparecimento de edemas faz parte dos cuidados de enfermagem ${ }^{3}$.

O diagnóstico de processos familiares interrompidos é gerado por uma alteração do estado de saúde de um membro familiar e tem como consequência a troca dos papéis na família, que são expressas nas mudanças da participação dos familiares na resolução de problemas e mudança na participação e tomada de decisões. Sendo a hantavirose uma doença que afeta significantemente pessoas do sexo masculino na maioria das vezes trabalhadores rurais, o processo se torna ainda mais doloroso, pois geralmente, o provedor da casa adoece ${ }^{24}$.

Além de todos os cuidados acima descritos existem outros que são fundamentais para garantir o conforto e a segurança do paciente no ambiente hospitalar para proporcionar uma melhor recuperação. Realizar o controle de infecção através de inúmeras precauções padrões é essencial para redução do risco de infecção vivenciado pelo paciente.

O paciente intubado perde suas barreiras naturais de defesa das vias aéreas 
superiores, além disso, a equipe através das suas mãos e do equipamento respiratório constitui a maior fonte de contaminação exógena ${ }^{25}$. Monitorar sinas e sintomas de infecção como edema, hiperemia, calor, rubor, hipertermia, realizar a higienização das mãos a cada procedimento com o paciente e fazer a utilização do gel alcoólico, promover a desinfecção com álcool a $70 \%$ nos dispositivos endovenosos antes de administrar medicações e utilizar técnica asséptica para realizar procedimentos são meios eficazes para reduzir os riscos de infecção ${ }^{20}$.

\section{CONCLUSÃO}

A sistematização da assistência de enfermagem desempenha um papel fundamental em descrever claramente as contribuições da Enfermagem no cenário de cuidados à saúde, garantindo um atendimento organizado, ético, responsável e baseado em evidências científicas.

Em se tratando de doenças virais emergentes de alta letalidade como a hantavirose, o desenvolvimento de uma assistência de excelência é primordial e poderá colaborar para redução da mortalidade, uma vez que a velocidade de sua evolução clínica requer um cuidado de enfermagem eficaz que detecte alterações clínicas precocemente e possibilite intervenções resolutivas.

Acredita-se que a utilização das afirmativas contribua para disseminar a prática, conceitos e cuidados de enfermagem, proporcionando aos enfermeiros uma linguagem específica da área, que possibilite o estímulo para pesquisas futuras, influenciando na educação em enfermagem e políticas de saúde. Todas essas ações também permitirá uma melhoria na assistência, uma vez que o diagnóstico retrata as reais necessidades dos pacientes, e, após a sua identificação, o enfermeiro terá subsídios, por meio das intervenções de enfermagem, para identificar as ações necessárias à sua assistência.

Recomenda-se a continuidade do estudo para que as afirmativas de diagnósticos e intervenções de enfermagem sejam reavaliadas e ajustadas às peculiaridades dos pacientes, fomentando assim, a reavaliação e reconstrução de uma assistência de qualidade.

\section{REFERÊNCIAS}


1. Kruger DH, Figueiredo LTM, Song JW, Klempa B. Hantaviruses-Globally emerging pathogens. J clin virol. 2015; 64:128-36.

2. Vaheri A, Strandin T, Hepojoki J, Sironen T, Henttonen H, Mäkelä S, et al. Uncovering the mysteries of hantavirus infections. Nat rev microbiol. 2013; 11:539-50.

3. Lemos ERS, Silva MV 2013. In: Coura JR, Editor. Dinâmica das Doenças Infecciosas e Parasitárias. Rio de Janeiro: Guanabara Koogan; 2013.

4. Rosa EST, Medeiros DBA, Nunes MRT, Simith DB, Pereira AS, Elkhoury MR, et al. Pygmy Rice Rat as Potential Host of Castelo dos Sonhos Hantavirus. Emerg infect dis. 2011; $17(8): 1527-30$.

5. Terças ACP, Espinosa MM, Santos MA. Fatores associados ao óbito por síndrome cardiopulmonar por hantavírus em Mato Grosso, Brasil. In: Guimarães LV, Pignatti MG, Souza DPO. Saúde Coletiva: múltiplos olhares em pesquisa. Cuiabá: EdUFMT; 2012.

6. Cofen. Conselho Federal de Enfermagem. Resolução $n^{\circ}$ 358/2009: Dispõe sobre a Sistematização da Assistência de Enfermagem e a implementação do Processo de Enfermagem em Ambientes públicos ou privados, em que ocorre o cuidado do profissional de Enfermagem, e dá outras providências. Available from: http://mt.corens.portalcofen.gov.br/resolucao-cofen-3582009_726.html

7. Amarante LN, Rossetto AP, Schneider DG. Sistematização da assistência de enfermagem em unidade de terapia intensiva sustentada pela teoria de Wanda Horta. Rev Esc Enferm USP. 2009; 43(1):54-64.

8. Horta WA. Processo de Enfermagem. São Paulo: EPU, 1979.

9. NANDA. Diagnósticos de Enfermagem da NANDA: Definições e classificação 20152017/[NANDA Intenational]; organizadoras: T. Heather Herdman, Shigemi Kamitsuru. Porto Alegre: Artmed; 2015.

10. Conselho Internacional de Enfermeiros. Cipe Versão 1 - Classificação Internacional para a Prática de Enfermagem Versão 1.0. São Paulo: Algol, 2007. [Título original: International Classification for Nursing Practice - ICNP $®$ Version 1.0]

11. MacNeil A, Ksiazek TG, Rolli P. Hantavirus Pulmonary Syndrome, United States, 1993-2009. Emerg infect dis. 2011; 17(7):1195-201.

12. Martinez VP, Bellomo CM, Cacace ML, Suárez $P$, Bogni L, Padula PJ. Hantavirus Pulmonary Sidrome in Argentina, 1995-2008. Emerg infect dis. 2010; 16(12):1853-60.

13. Manigold T, Vial P. Human hantavirus infections: epidemiology, clinical features, pathogenesis and immunology. Swiss med wkly. 2014; 144:w13937. 
14. Krüger DH, Schönrich $G$, Klempa B. Human pathogenic hantaviruses and prevention of infection. Hum Vaccin. 2011; 7(6):685-93.

15. Oliveira SV, Fonseca LX, Silva PMRB, Pereira SVC, Caldas EP. Análise do perfil epidemiológico da hantavirose no Brasil no período de 2007 a 2012. Rev patol trop. 2014; 43(2):131-42.

16. Santos 10, Figueiredo GG, Figueiredo LTM, Azevedo MRA, Novo NF, Vaz CAC. Serologic survey of Hantavirus in a rural population from the northern State of Mato Grosso, Brazil. Rev Soc Bras Med Trop. 2013; 46(1):30-3.

17. Spiropoulou CF, Srikiatkhachorn A. The role of endothelial activation in dengue hemorrhagic fever and hantavirus pulmonary syndrome. Virulence. 2013; 4(6): 525-36.

18. Nascimento VF, Maciel MM, Lemes AG, Borges AP, Terças ACP, Hattori TY. Percepções de familiares sobre hospitalização no ambiente intensivo. Rev Enferm UFPI. 2015; 4(2):92-9.

19. Nascimento VF, Maciel MM, Terças ACP, Lemes AG, Hattori TY, Nascimento VF, et al. Apreensões e sentimento de fé de familiares no ambiente de cuidado intensivo. Revista Eletrônica Gestão \& Saúde. 2015; 6(3):2639-55.

20. Oliveira AC, Kovner CT, Silva RS. Infecção hospitalar em unidade de tratamento intensivo de um hospital universitário brasileiro. Rev latinoam enferm. 2010; 18(2):08 telas.

21. Paes GO, Mello ECP, Leite JL, Mesquita MGR, Oliveira FT, Carvalho SM. Protocolo de cuidados ao cliente com distúrbio respiratório: ferramenta para tomada de decisão aplicada à enfermagem. Esc Anna Nery. 2014; 18(2): 303-10.

22. Ramos CCS, Dal Sasso GTM, Martins CR, Nascimento ER, Barbosa SFF, Martins JJ, et al. Monitorização hemodinâmica invasiva a beira do leito: avaliação e protocolo de cuidados de enfermagem. Rev Esc Enferm USP. 2008; 42(3):512-8.

23. Mackow E R, Gorbunova E E and Gavrilovskaya I N Endothelial cell dysfunction inviral hemorrhage and edema. Front Microbiol. 2015; 5:733.

24. Montefusco SRA. Diagnósticos de enfermagem identificados em famílias em situação de acompanhamento hospitalar utilizando o modelo Calgary. Rev eletrônica enferm. 2008;10(1):254-6.

25. Favretto DO, Silveira RCCP, Canini SRMS, Garbin LM, Martins FTM, Dalri MCB. Aspiração endotraqueal em pacientes adultos com via aérea artificial: revisão sistemática. Rev Latinoam enferm. 2012; 20(5):10 telas. 
Conflito de interesses: Os autores declaram não haver conflito de interesses.

Participação dos autores: Os autores declaram que participaram de todas as etapas do estudo (concepção, desenvolvimento do estudo, redação e revisão).

Como citar este artigo: Terças ACP, Souza IG, Moura ACP, Nascimento VF, Hattori TY, Gleriano JS, et al. Sistematização da assistência de enfermagem no monitoramento clínico de pacientes com hantavirose. Journal Health NPEPS. 2017; 2(2):391-406. 